\title{
A case report of SMARCA4/BRG1-deficient non- small cell lung cancer with a spindle cell component found by next-generation sequencing of a brain metastasis
}

Hiroko Ikeda ( $\sim$ h-ikeda@med.kanazawa-u.ac.jp )

Kanazawa University Hospital https://orcid.org/0000-0001-7105-6376

\section{Takashi Sone}

Kanazawa University

Kazuo Kasahara

Kanazawa University

\section{Satoko Nakada}

Kanazawa University Hospital

Kaori Yoshimura

Kanazawa University Hospital

\section{Maki Sakaguchi}

Kanazawa University Hospital

\section{Keishi Mizuguchi}

Kanazawa University Hospital

\section{Takayuki Nojima}

Kanazawa University Hospital

Shinji Takeuchi

Kanazawa University

\section{Seiji Yano}

Kanazawa University

Kotaro Yoshida

Kanazawa University Graduate School of Medicine

Toshifumi Gabata

Kanazawa University Graduate School of Medicine

\section{Case Report}

Keywords: BRG1; SMARCA4; Non-small cell lung cancer; Thoracic sarcoma; Case report

Posted Date: July 29th, 2020 
DOI: https://doi.org/10.21203/rs.3.rs-45392/v1

License: (c) (i) This work is licensed under a Creative Commons Attribution 4.0 International License. Read Full License 


\section{Abstract}

Background: Several studies of different cancers have revealed mutations in switch/sucrose nonfermenting (SWI/SNF) complex genes. Brahma-related gene 1 (BRG1), which is encoded by SWI/SNFrelated, matrix-associated, actin-dependent regulator of chromatin, subfamily $\mathrm{A}$, member 4 (SMARCA4), is a member of this complex. SMARCA4/BRG-1-deficient non-small cell lung carcinoma (NSCLC) has been considered a subset of lung cancer that has distinct clinical, pathological, and molecular characteristics, which implies its relationship with SMARCA4/BRG1-deficient thoracic sarcoma.

Case presentation: We experienced a case of SMARCA4/BRG1-deficient lung cancer in a 40-year-old female patient with a history of smoking. Magnetic resonance imaging revealed a large mass in the right lung apex with an extension to the extrapulmonary region and cerebral metastasis. Histological analysis showed poorly differentiated carcinoma with spindle cell components. She was diagnosed with NSCLC (stage IV) at that point. An EGFR mutation and ALK and ROS1 rearrangement were not detected, and then treated with chemoradiotherapy. Overall, the tumors were resistant to chemotherapy, and therefore, after 2 years, the brain tumor was excised for histological and molecular analysis. Histologically, the brain mass was an undifferentiated tumor with round cells and glandular components. The mutation in SMARCA4 in the brain specimen was identified by next-generation sequencing. Immunohistochemical examination revealed a complete loss of BRG1. SMARCA4/BRG1-deficient thoracic sarcoma had been raised as a differential diagnosis, collectively, she was diagnosed with SMARCA4/BRG1-deficient NSCLC considering for the result of positivity for cytokeratin AE1/AE3 and claudin-4, and negativity for Sal-like protein 4, CD34, and SRY-box 2 by immunohistochemical examination. Regrettably, a definitive diagnosis required approximately 2 years. She is alive with disease at 30 months after the presentation.

Conclusions: The diagnosis of SMARCA4/BRG1-deficient NSCLC is frequently difficult because of no specific morphology and necessity of discrimination from SMARCA4/BRG1-deficient thoracic sarcoma, which is the practical reason this disease is sometimes missed. Immunohistochemistry for BRG1 should be encouraged for the pathological examination of NSCLC with any histology for the prompt and precise diagnosis of SMARCA4/BRG1-deficient NSCLC.

\section{Background}

The switch/sucrose non-fermenting (SWI/SNF) complex is an evolutionarily conserved ATPasedependent chromatin remodeling complex that plays important roles in gene expression, transcription, differentiation, and DNA repair. Each complex is composed of a mutually exclusive ATP-dependent catalytic unit, either Brahma-related gene 1 (BRG1), which is encoded by SWI/SNF-related, matrixassociated, actin-dependent regulator of chromatin, subfamily $\mathrm{A}$, member 4 (SMARCA4), or Brahma (BRM), which is encoded by SWI/SNF-related, matrix-associated, actin-dependent regulator of chromatin, subfamily A, member 2 (SMARCA2), and a variable number of core subunits collectively referred to as BRG1/BRM-associated factors (BAFs). Integrase interactor (INI-1), which is encoded by SWI/SNF-related, matrix-associated, actin-dependent regulator of chromatin, subfamily $\mathrm{B}$, member 1 (SMARCB1), is also 
part of the BAF complex [1]. Several studies of different cancer types have reported gene mutations in BAFs, which suggests that this complex has a tumor suppressor function. Malignant rhabdoid tumors and pediatric atypical teratoid/rhabdoid tumors are characterized by the loss of INI-1 expression in the majority of cases, whereas a few cases in which BRG1 is lost maintain SMARCB1 expression. Almost all epithelioid sarcomas lack INI-1 expression, and the somatic or germline alterations in the SMARCA4 gene are highly associated with small cell carcinoma of the ovary, hypercalcemic type [2-5].

SMARCA4/BRG1 loss in carcinomas has been reported in several organs including the lungs.

SMARCA4/BRG1 loss occurs in approximately $4-10 \%$ of lung carcinomas, in which the histology was primarily poorly differentiated non-small cell lung carcinoma (NSCLC) including adenocarcinoma, mucinous carcinoma, squamous cell carcinoma, large cell carcinoma, and pleomorphic carcinoma. SMARCA4/BRG-1-deficient has been considered a subset with a heterogeneous spectrum because of its morphological features, such as solid growth and lack of a lepidic pattern; its negativity for thyroid transcription factor 1 (TTF-1) by immunohistochemistry; lack of actionable gene alterations, such as epidermal growth factor receptor ( $E G F R)$ mutations and anaplastic lymphoma kinase ( $A L K)$ and protooncogene 1 (ROS1) fusions; and poor prognosis [6-10]. However, pathologists might not note the precise diagnosis if they are unable to recognize SMARCA4/BRG-1-deficient NSCLC, including the ability to discriminate this from other SMARCA4/BRG1-deficient thoracic neoplasms represented by SMARCA4deficient thoracic sarcoma. Here, we report a case of SMARCA4/BRG1-deficient lung cancer with a sarcomatous component, in which BRG1 loss was detected by next-generation sequencing (NGS) of a brain metastasis specimen.

\section{Case Presentation}

\section{Clinical Summary}

A Japanese woman in her early 40 s without any history of malignancy presented to our hospital with complaints of worsening pain in the back, chest, and arm, as well as further paralysis of the right hand. She had a medical history of rheumatoid arthritis and Sjogren syndrome and a history of smoking (Brinkman index: 300). Computed tomography (CT) and magnetic resonance imaging (MRI) revealed bullae and a mass in the right lung apex approximately $10 \mathrm{~cm}$ in diameter with extension to the mediastinum, clavicle, costal, vertebra, and brachial plexus; a concurrent mass was also observed in the right occipital lobe (Fig. 1). Radiologists considered the lung to be the primary tumor site. CT-guided needle biopsy was performed for the primary tumor, and thereafter, emergency decompression against the vertebral tumor was performed. The pathological diagnosis of the primary tumor was poorly differentiated carcinoma and was suspected to be a sarcomatoid carcinoma. She was diagnosed with NSCLC (stage IV) at that point. An EGFR mutation and ALK and ROS1 rearrangement were not detected. The PD-L1 tumor proportion score was $70-80 \%$. She was then treated with cisplatin + tegafur, atezolizumab, docetaxel + ramucirumab, vinorelbine, pemetrexed, pembrolizumab, and radiation therapy for the thoracic tumor and underwent $y$-knife treatment for the brain metastasis. Overall, the tumors were resistant to chemotherapy, and therefore, after 2 years, the brain tumor was excised for histological and 
molecular analysis. Histologically, the brain mass was an undifferentiated tumor with round cells and glandular components. The mutation in SMARCA4 in the brain specimen was identified in formalin-fixed paraffin-embedded material by NGS. Immunohistochemical examination revealed complete BRG1 loss in tumor cells. Collectively, she was diagnosed with SMARCA4/BRG1-deficient non-small cell lung cancer. Among the tumor serum markers analyzed, she exhibited no hypercalcemia and only mild elevation of CYFRA (cytokeratin 19). She is currently alive with disease at 30 months after the presentation.

\section{Pathological Findings}

CT-guided needle biopsy revealed a poorly differentiated carcinoma with malignant spindle cells. Histologically, irregular noncohesive spindle cells had infiltrated the lung parenchyma and pleura, and polygonal cells with pyknotic nuclei and eosinophilic cytoplasm were scattered or arranged in a trabecular pattern in the fibrous/hyalinous stroma (Fig. 2a, b). Glandular, squamous, neuroendocrine, and specific mesenchymal differentiation, such as the presence of rhabdoid features, was not evident. Immunohistochemically, tumor cells were diffusely positive for cytokeratin (CK) AE1/AE3 (Fig. 2c). Nuclear staining for TTF-1 was not observed, whereas p40 staining was observed in a few nuclei (Fig. 2d, e). Bizarre, large cells with prominent nuclear atypia and occasional small vesicles in an alveolar pattern were found in the vertebral specimen (Fig. 2g, h). The brain tumor showed solid growth of dyscohesive round cells with well-delineated cell borders as the primary histology, but occasionally, cells arranged in a sheet-like pattern were seen (Fig. 3a). Spindle or polygonal neoplastic cells observed in the myxoid stroma, which resembled a thoracic tumor, were only a minor component of the brain metastasis

(Fig. 3b). Mitotic figures and extensive necrosis were both clearly seen. Pseudo-papillary architecture with hobnail cells and isolated rhabdoid-like cells were mixed in some areas (Fig. 3c). Some emperipolesis associated with granulocytes was observed (Fig. 3d). Goblet-like cells and intracytoplasmic mucin were visible by Alcian blue staining (Fig. 3e). Immunohistochemical analysis revealed the diffuse expression of CK AE1/AE3, the focal expression of claudin-4, and scarce expression of p40 (Fig. 3g, h, i). The histology of the brain metastasis was mainly poorly/undifferentiated carcinoma with features of an adenocarcinoma. We performed immunostaining for BRG1 after the detection of a SMARCA4 mutation in the brain specimen by NGS, and BRG1 loss was demonstrated in the brain tumor as well as in the lung and vertebral tumors (Fig. 2f, i, 3f). Sal-like protein 4 (SALL4), CD34, SRY-box 2 (SOX2), p53, calretinin, NUT, and CD 5 were consistently negative in every specimen. BRM loss was found to varying degrees. The NCC Oncopanel System identified a loss-of-function mutation at Q1012 (3034C > T) in SMARCA4. Finally, a diagnosis of SMARCA4-deficient non-small lung cell cancer was made. The TP3 and KDM6A genes were also observed to contain actionable alterations. The tumor mutational burden (TMB) was 49.6/Mb, which was considered high.

\section{Discussion And Conclusions}

We experienced the case of a young female with SMARCA4/BRG1-deficient lung cancer. Regrettably, a definitive diagnosis required approximately 2 years. The most frequent histology of SMARCA4/BRG1- 
deficient lung cancer has been reported to be solid adenocarcinoma, large cell carcinoma, and pleomorphic carcinoma with occasional rhabdoid features $[6,7,10]$. In our case, the major histological pattern was poorly differentiated carcinoma with malignant spindle cells in abundant hyalinous stroma according to the CT-guided needle biopsy; however, these morphological features have been seldom described in articles about SMARCA4/BRG1-deficient NSCLC. Although the undifferentiated round cell morphology and rhabdoid features seen in SMARCA4/BRG1-deficient neoplasms seem to be notable characteristics in several organs $[5,11,12]$, the loss of SMARCA4/BRG1 has been found in more welldifferentiated adenocarcinomas but not in squamous cell carcinoma of the lung $[7,10]$. Immunohistochemistry for BRG1 in NSCLC of each histologic type should be performed not only to note SMARCA4/BRG1 loss but also because SMARCA4/BRG1-deficient NSCLC has been considered a peculiar subset with aggressive clinical behavior and the potential to benefit from targeted therapy. Differential diagnoses would be presented at that time and would include SMARCA4/BRG1-deficient thoracic neoplasms and lung metastasis of another SWI/SNF complex-related malignant neoplasm. Immunohistochemistry for BRG1 has been thought to have sufficient sensitivity and specificity for the diagnosis of SMARCA4/BRG1-deficient thoracic neoplasms [7, 10], although additional examination of SMARCA4 gene alterations ensures the diagnosis.

Radiologists suspected that the origin of the tumor in this case was the lung, but the tumor was distributed in the right lung apex, mediastinum, chest wall, and vertebra. Therefore, the possibility of mesothelioma, thymic carcinoma, and NUT carcinoma should be raised as differential diagnoses; moreover, SMARCA4/BRG1-deficient thoracic sarcoma should not be disregarded. The loss of SMARCA4/BRG1 was reported in some cases of mesothelioma [13] but has not been shown in thymic carcinoma and NUT carcinoma. Our case was negative for calretinin, CD5, and NUT by immunohistochemistry, and thus, these cancers were unlikely.

SMARCA4/BRG1-deficient thoracic sarcoma has been recently recognized as a peculiar disease with characteristics such as affected patients who are relatively young with a history of heavy smoking and emphysema/bullae in the lungs, the presence of large thoracic tumors, poor prognosis, and undifferentiated dyscohesive round cell morphology with rhabdoid features. We observed similar findings in our patients, such as younger age, smoking history, large thoracic mass in a bullous background, and poorly differentiated round cell morphology of the brain metastasis. We compared the findings of our case with those of SMARCA4/BRG1-deficient thoracic sarcoma/NSCLC. The results of this comparison are shown in Table $1[7,9,10,14-18]$. We judged that our case was likely SMARCA4/BRG1-deficient NSCLC rather than a thoracic sarcoma considering the immunohistochemistry results, i.e., claudin-4 positivity and SOX2, CD34, and SALL4 negativity. Emperipolesis was observed in the brain metastasis in our case, which was reported in a previous article on SMARCA4/BRG1-deficient NSCLC [7], but this has not yet been described in SMARCA4/BRG1-deficient thoracic sarcoma. Emperipolesis in the tumor may be a clue that can be used for discrimination. 
Table 1

Comparative analysis of the clinicopathological and genetic findings of SMARCA4/BRG1-deficient thoracic tumors including both the present case and published cases

SMARCA4/BRG1-deficient thoracic sarcoma
SMARCA4/BRG1deficient NSCLC

Present case

Clinical feature

History of

smoking, $\mathrm{n}(\%)$

$48 / 55(87.2 \%)$

$14 / 17(82.4 \%)$

Present

Primary tumor

location ( $n, \%)$

Abdominal cavity $(1 / 12,8.3 \%)$, axilla

$(1 / 12,8.3 \%)$, chest wall $(12 / 49,24.5 \%)$,

Lung $(37 / 37,100 \%)$

diaphragm $(6 / 72,8.3 \%)$, esophagus

$(2 / 36,5.6 \%)$, heart $(1 / 17,5.9 \%)$, lung

$(38 / 60,63.3 \%)$, mediastinum (35/41, $85.4 \%)$, pelvis $(1 / 12,8.3 \%)$, pericardium

$(2 / 19,10.5 \%)$, retroperitoneum $(1 / 12$,

$8.3 \%)$, pleura $(16 / 36,44.4 \%)$, vertebra

$(4 / 33,12.1 \%)$

Site of

metastasis $(\mathrm{n}$,

$\%)$
Adrenal gland $(11 / 50,22 \%)$, abdominal cavity $(1 / 19,5.3 \%)$, bone $(14 / 49,28.6 \%)$, brain $(1 / 19,5.3 \%)$, chest wall $(1 / 17$, $5.9 \%)$, dura $(2 / 19,10.5 \%)$, esophagus $(1 / 17,5.9 \%)$, kidney $(3 / 36,8.3 \%)$, liver $(11 / 53,20.8 \%)$, lung $(8 / 29,27.6 \%)$, lymph node (23/50, 46\%), mesentery $(2 / 36,5.6 \%)$, omentum $(1 / 17,5.9 \%)$, ovary $(1 / 12,8.3 \%)$, pancreas $(2 / 17$, $11.8 \%)$, peritoneum/retroperitoneum $(6 / 36,16.7 \%)$, pleura $(3 / 29,10.3 \%)$, stomach $(4 / 29,13.8 \%)$, soft tissue $(2 / 29$, $6.9 \%)$, vertebra $(7 / 29,24.1 \%)$
Abdominal wall
$(1 / 18,5.5 \%)$, adrenal gland $(5 / 18$, $27.8 \%)$, bone $(4 / 18$, $22.2 \%)$, brain $(2 / 18$, $11.1 \%)$, liver (4/18, $22.2 \%)$, lung (3/19, $15.8 \%)$, lymph node (4/18. 22.2\%), peritoneum (3/18, $16.7 \%)$, pleura
$(5 / 18,27.8 \%)$, skin
$(1 / 18,5.5 \%)$
Brain

Emphysema and/or bullae, $\mathrm{n}$ $33 / 49$ (67.3\%) Bullae

Lung, mediastinum, chest wall, vertebra, clavicle, brachial plexus

(\%)

Histology

Undifferentiated

round cell, $n$ (\%)

Rhabdoid cell, $\mathrm{n}$

(\%)

Spindle cell, $\mathrm{n}$

(\%)
$52 / 52(100 \%)$

$23 / 52(44.2 \%)$

$2 / 14(14.3 \%)$
$4 / 36(11.1 \%)$

Present

$5 / 36(13.9 \%)$

Present

$1 / 40(2.5 \%)$

Present

Abbreviations: ALK, anaplastic lymphoma kinase; BAF, BRG1/BRM-associated factors; BRG1, Brahmarelated gene 1; BRM, Brahma; CT, computed tomography; CK, cytokeratin; EGFR, epidermal growth factor receptor; IHC, immunohistochemistry; NSCLC, non-small cell lung carcinoma; ROS1, ROS protooncogene 1; SALL4, Sal-like protein 4; SMARCA4, SWI/SNF-related, matrix-associated, actindependent regulator of chromatin, subfamily A, member 4; SOX2, SRY-box 2; TTF-1, thyroid transcription factor 1. 


\section{SMARCA4/BRG1-deficient thoracic sarcoma}

SMARCA4/BRG1deficient NSCLC

Non-small cell carcinoma, $\mathrm{n}$

$2 / 32(6.3 \%)$

39/41 (95.1\%)

Present

(\%)

IHC

\begin{tabular}{|lccl|}
$\begin{array}{l}\text { BRG1 lost or } \\
\text { reduced, } \mathrm{n}(\%)\end{array}$ & $52 / 52(100 \%)$ & $41 / 41(100 \%)$ & $\begin{array}{l}\text { Negative } \\
\text { (lost) }\end{array}$ \\
\hline $\begin{array}{l}\text { BRM lost or } \\
\text { reduced, } \mathrm{n}(\%)\end{array}$ & $43 / 46(93.5 \%)$ & $23 / 85(27.1 \%)$ & $\begin{array}{l}\text { Present } \\
\text { (reduced) }\end{array}$ \\
$\begin{array}{l}\text { CK positivity, n } \\
(\%)\end{array}$ & $28 / 52(53.8 \%)$ & $23 / 24(95.8 \%)$ & Positive \\
\hline
\end{tabular}

(\%)

TTF-1 positivity $\quad 5 / 49(10.2 \%)$

11/41 (26.8\%) Negative

p40 positivity $\quad$ 2/39 (5.1\%)

$2 / 18(11.1 \%)$

Positive, a

few

Claudin-4

$0 / 27(0 \%)$

$56 / 52(100 \%)$

Positive, positivity $30 / 32$ (93.8\%)

$4 / 16(25 \%)$ focal

SOX2 positivity
$31 / 52(59.6 \%)$

$0 / 17(0 \%)$

Negative

CD34 positivity

$19 / 32(59.4 \%)$

$5 / 78(6.4 \%)$

Negative

SALL4

positivity

p53 positivity

10/14 (71.4\%)

$2 / 2(100 \%)$

Negative

Genetic alterations

\begin{tabular}{llll|} 
SMARCA4 & $38 / 40(95 \%)$ & $14 / 17(82.4 \%)$ & Positive \\
\hline EGFR & $0 / 9(0 \%)$ & $0 / 25(0 \%)$ & Negative \\
\hline ALK/ROS1 & $0 / 9(0 \%)$ & $0 / 17(0 \%)$ & Negative \\
\hline TP53 & $27 / 33(81.8 \%)$ & $15 / 17(88.2 \%)$ & Positive \\
\hline KRAS & $3 / 20(15 \%)$ & $6 / 21(28.6 \%)$ & Negative \\
\hline STK11 & $5 / 20(25 \%)$ & $3 / 12(25 \%)$ & Negative \\
KEAP1 & $3 / 20(15 \%)$ & & Negative \\
\hline
\end{tabular}

Abbreviations: ALK, anaplastic lymphoma kinase; BAF, BRG1/BRM-associated factors; BRG1, Brahmarelated gene 1; BRM, Brahma; CT, computed tomography; CK, cytokeratin; EGFR, epidermal growth factor receptor; IHC, immunohistochemistry; NSCLC, non-small cell lung carcinoma; ROS1, ROS protooncogene 1; SALL4, Sal-like protein 4; SMARCA4, SWI/SNF-related, matrix-associated, actindependent regulator of chromatin, subfamily A, member 4; SOX2, SRY-box 2; TTF-1, thyroid transcription factor 1. 
The absolute discrimination between SMARCA4/BRG1-deficient thoracic sarcoma and SMARCA4/BRG1deficient NSCLC is difficult from a clinicopathologic perspective and also because of their genetic similarities; specifically, a thoracic mass may be distributed from the lung to the mediastinum/chest wall. Nowadays, alterations in SWI/SNF chromatin-remodeling complex genes, such as those in SMARCB1, SMARCA2, SMARCA4, and ARID1A, are believed to be associated with dedifferentiation of the carcinoma and epithelial-mesenchymal transition in several organs $[11,12,15]$. Rekhtman et al. [16] proposed that SMARCA4/BRG1-deficient thoracic sarcoma represents undifferentiated/dedifferentiated lung carcinoma associated with smoking history, which demonstrates the presence of smoking-related genetic alterations in the component of the NSCLC juxtaposed sarcomatoid area in SMARCA4/BRG1-deficient thoracic sarcoma. Dedifferentiated SMARCA4/BRG1-deficient NSCLC would likely be present in patients diagnosed with SMARCA4/BRG1-deficient thoracic sarcoma, but conversely, SMARCA4/BRG1-deficient thoracic sarcoma might be considered to be undifferentiated lung carcinoma. We wonder whether pure SMARCA4/BRG1-deficient thoracic sarcoma, which would be located only in the mediastinum and contain no lung lesions or epithelial elements, is a unique entity and whether there is an intermediate tumor type between thoracic sarcoma and dedifferentiated carcinoma that is yet to be defined (Fig. 4).

In this case, the alteration in SMARCA4 was located on exon22:c.3034C > T, Q1012, and was a stop-gain single nucleotide variant type, which is considered a truncating mutation. The same codon mutation in SMARCA4 was reported by Yoshida et al. ${ }^{15}$ in a patient with SMARCA4/BRG1-deficient thoracic sarcoma, who was a 40 year-old male smoker treated with surgery and chemotherapy but died after 7 months. His primary tumor was located in the axilla, and the metastatic sites were the lymph node, adrenal gland, and stomach. Remarkably, the lung lesion was not substantial in size during the disease period. An immunohistochemical analysis of his tumor revealed BRG1 loss, BRM positivity, CK AE1/AE3 positivity, CD34/SOX2/SALL4 negativity, and claudin-4 negativity. The diagnosis of SMARCA4/BRG1-deficient thoracic sarcoma was appropriate for that case; however, there were unusual findings, such as no bullous background, retention of BRM, and negativity of stem cell markers (CD34/SOX2/SALL4). These two cases with the same genetic alteration in SMARCA4 that also shared some similar clinical and immunohistochemical features were different in that one case was classified as SMARCA4/BRG1deficient thoracic sarcoma, and the other was classified as SMARCA4/BRG1-deficient NSCLC; these diagnoses were made regardless of whether a lung mass, different carcinoma components, or the expression of epithelial markers, such as CK and claudin-4, was observed or not. We surmise that a relationship between SMARCA4/BRG1-deficient thoracic sarcoma and SMARCA4/BRG1-deficient NSCLC exists and might be influenced by the status of other factors including another SWI/SNF complex component (BRM) or TP53 $[10,15,16]$.

In this case, the histology was not identical between the primary thoracic mass and the metastatic brain lesion, which was resected 2 years after presentation. Interestingly, the histological findings were more typical of a SMARCA4/BRG1-deficient neoplasm, such as a brain metastasis, than a lung tumor. The histological difference may represent some degree of dedifferentiation, or a peculiarity in each organ; likewise, an atypical teratoid/rhabdoid tumor of the central nervous system also frequently exhibits 
undifferentiated round cell tumor morphology with rhabdoid features, although the first biopsy specimen might represent a small portion of a larger heterogeneous tumor.

As molecular technology progresses, the detection of key gene mutations by NGS will increase such as in this case. An effective treatment of SMARCA4-deficient NSCLC has not yet been established. Recently, high TMB in SMARCA4/BRG1-deficient thoracic sarcoma/NSCLC was also detected by NGS, which suggests the possibility of a treatment strategy based on immune checkpoint inhibitors $[8,9]$.

In conclusion, we experienced a case of SMARCA4/BRG1-deficient NSCLC, which typically presents in young patients who are smokers and usually presents in a bullous background as a large tumor in the lung apex, along with aggressive behavior, and requires distinction from SMARCA4/BRG1-deficient thoracic sarcoma. SMARCA4/BRG1-deficient NSCLC has no specific morphology, which is the practical reason this disease is sometimes missed. Immunohistochemistry for BRG1 should be encouraged for the pathological examination of NSCLC with any histology, except for adenocarcinoma with a lepidic pattern. SMARCA4/BRG1-deficient NSCLC lacks targetable alterations in EGFR/ALK/ROS1, and therefore, further investigation is necessary to discover the potential of therapeutic personalization and improvement in prognosis.

\section{Abbreviations}

ALK: anaplastic lymphoma kinase; BAFs: BRG1/BRM-associated factors; BRG1: Brahma-related gene 1; BRM: Brahma; CT: computed tomography; CK: cytokeratin; EGFR: epidermal growth factor receptor; IHC: immunohistochemistry; INI-1: integrase interactor; MRI: magnetic resonance imaging; NGS: nextgeneration sequencing; NSCLC: non-small cell lung carcinoma; ROS1: ROS proto-oncogene 1; SALL4: Sallike protein 4; SMARCB1: SWI/SNF-related, matrix-associated, actin-dependent regulator of chromatin, subfamily B, member 1; SMARCA2: SWI/SNF-related, matrix-associated, actin-dependent regulator of chromatin, subfamily A, member 2; SMARCA4: SWI/SNF-related, matrix-associated, actin-dependent regulator of chromatin, subfamily A, member 4; SOX2: SRY-box 2; SWI/SNF: switch/sucrose nonfermenting complex; TMB: tumor mutational burden; TTF-1: thyroid transcription factor 1.

\section{Declarations}

\section{Acknowledgments}

The authors gratefully acknowledge the work of the members of Diagnostic Pathology, Kanazawa University Hospital for helpful discussions and comments on the manuscript.

\section{Authors' contributions}

$\mathrm{HI}$ performed the histological evaluation and wrote the manuscript; TS and KK evaluated the clinical data; ST and SY performed the genetic analysis; SN, KY, MS, and KM performed the histological evaluation; KY and TG evaluated the radiological data. 


\section{Funding}

The authors have no funding to disclose.

\section{Availability of date and materials}

All date generated or analyzed during this study are included in this published article.

\section{Ethics approval and consent to participate}

The patient provided consent for publication of the case report.

\section{Consent for publication}

Written informed consent was obtained from the family of the patient for the publication of this case report.

\section{Competing interests}

The authors declare the they have no competing interests.

\section{Author details}

${ }^{1}$ Diagnostic Pathology, Kanazawa University Hospital, 13-1, Takaramachi, Kanazawa-City, Ishikawa, 8641-920, Japan. ${ }^{2}$ Department of Respiratory Medicine, Kanazawa University, 13-1, Takaramachi, Kanazawa-City, Ishikawa, 8640-920, Japan. ${ }^{3}$ Division of Medical Oncology, Cancer Research Institute, Kanazawa University, 13-1, Takaramachi, Kanazawa-City, Ishikawa, 8641-920, Japan. ${ }^{4}$ Department of Radiology, Kanazawa University Graduate School of Medicine, 13-1, Takaramachi, Kanazawa-City, Ishikawa, 8640-920, Japan.

\section{References}

1. Kadoch C, Crabtree GR. MammalianSWI/SNF chromatin remodeling complexes and cancer: Mechanistic insights gained from human genomics. Sci Adv. 2015;1:e1500447.

2. Jackson EM, Sievert AJ, Gai X, Hakonarson H, Judkins AR, Tooke L, et al. Genomic analysis using high-density single nucleotide polymorphism-based oligonucleotide arrays and multiplex ligationdependent probe amplification provides a comprehensive analysis of INI1/SMARCB1 inmalignant rhabdoid tumors. Clin Cancer Res. 2009;15:1923-30.

3. Hasselblatt M, Gesk S, Oyen F, Rossi S, Viscardi E, Giangaspero F, et al. Nonsense mutation and inactivation of SMARCA4 (BRG1) in an atypical teratoid/rhabdoid tumor showing retained SNARCB1 (INI1) expression. Am J Surg Pathol. 2011;35:933-5.

4. Hornic JL, Dal Cin P, Fletcher CD. Loss of INI 1 expression is characteristic of both conventional and proximal-type epithelioid sarcoma. Am J Surg Pathol. 2009;33:542-50. 
5. Conlon N, Silva A, Guerra E, Jelinic P, Schlappe BA, Olvera N, et al. Loss of SMARCA4 expression is both sensitive and specific for the diagnosis of small cell carcinoma of ovary, hypercalcemic type. Am J Surg Pathol. 2016;40:395-403.

6. Yoshimoto T, Matsubara D, Nakano T, Tamura T, Endo S, Sugiyama Y, et al. Frequent loss of the expression of multiple subunits of the SWI/SNF complex in large cell carcinoma and pleomorphic carcinoma of the lung. Pathol Int. 2015;65:595-602.

7. Agaimy A, Fuchs F, Moskalev EA, Sirbu H, Hartmann A, Haller F. SMARCA4-deficientpulmonary adenocarcinoma: clinicopathological, immunohistochemical, and molecular characteristics of a novel aggressive neoplasm with a consistent TTF $1_{\text {neg }} / \mathrm{CK} 7_{\text {pos }} /$ HepPar- $1_{\text {pos }}$ Virchows Arch. 2017;471:599-609.

8. Naito T, Udagawa H, Umemura S, Sakai T, Zenke Y, Kirita K, et al. Non-small cell lung cancer with loss of expression of theSWI/SNF complex is associated with aggressive clinicopathological features, PD-L1-positive status, and high tumor mutation burden.Lung Cancer. 2019;138:35-42.

9. Naito T, Umemura S, Nakamura H, Zenke Y, Udagawa H, Kirita K, et al. Successful treatment with nivolumab forSMARCA4-deficient non-small cell lung carcinoma with a high tumor mutation burden: A case report.Thorac Cancer. 2019;10:1285-8.

10. Nambirajan A, Singh V, Bhardwaj N, Mittal S, Kumar S, Jain D. SMARCA4/BRG1-Deficient Non-Small CellLung Carcinomas: A Case Series and Review of the Literature. Arch Pathol Lab Med. 2020. doi: 10.5858/arpa.2019-0633-OA.

11. Agaimy A, Daum O, Märkl B, Lichtmannegger I, Michal M, Hartmann A. SWI/SNFComplex-deficient Undifferentiated/Rhabdoid Carcinomas of the Gastrointestinal Tract: A Series of 13 Cases Highlighting Mutually Exclusive Loss of SMARCA4 and SMARCA2 and Frequent Co-inactivation of SMARCB1 and SMARCA2.Am J Surg Pathol. 2016;40:544-53.

12. Karnezis AN, Hoang LN, Coatham M, Ravn S, Almadani N, Tessier-Cloutier B, et al. Loss of switch/sucrose non-fermenting complex protein expression is associated with dedifferentiation in endometrial carcinomas.Mod Pathol. 2016;29:302-14.

13. Perret R, Chalabreysse L, Watson S, Serre I, Garcia S, Forest F, et al. SMARCA4-deficient Thoracic Sarcomas: Clinicopathologic Study of 30 Cases With an Emphasis on Their Nosology and Differential Diagnoses. Am J Surg Pathol. 2019;43:455-65.

14. Le Loarer F, Watson S, Pierron G, de Montpreville VT, Ballet S, Firmin N, et al. SMARCA4 inactivation defines a group of undifferentiated thoracic malignancies transcriptionally related to BAF-deficient sarcomas. Nat Genet. 2015;75:2264-71.

15. Yoshida A, Kobayashi E, Kubo T, Kodaira M, Motoi T, Motoi N, et al. Clinicopathological and molecular characterization of SMARCA4-deficient thoracic sarcomas with comparison to potentially related entities. Mod Pathol. 2017;30:797-809.

16. Rekhtman N, Montecalvo J, Chang JC, Alex D, Ptashkin RN, Ai N, et al. SMARCA4DeficientThoracic Sarcomatoid Tumors Represent Primarily Smoking-Related Undifferentiated Carcinomas Rather Than Primary Thoracic J Thorac Oncol. 2020;15:231-47. 
17. Kunimasa K, Nakamura H, Sakai K, Tamiya M, Kimura M, Inoue T, et al. Patients withSMARCA4deficient thoracic sarcoma and severe skeletal-related events.Lung Cancer. 2019;132:59-64.

18. Kuwamoto S, Matsushita M, Takeda K, Tanaka N, Endo Y, Yamasaki A, et al. SMARCA4-deficient thoracicsarcoma: report of a case and insights into how to reach the diagnosis using limited samples and resources. Hum Pathol. 2017;70:92-7.

\section{Figures}

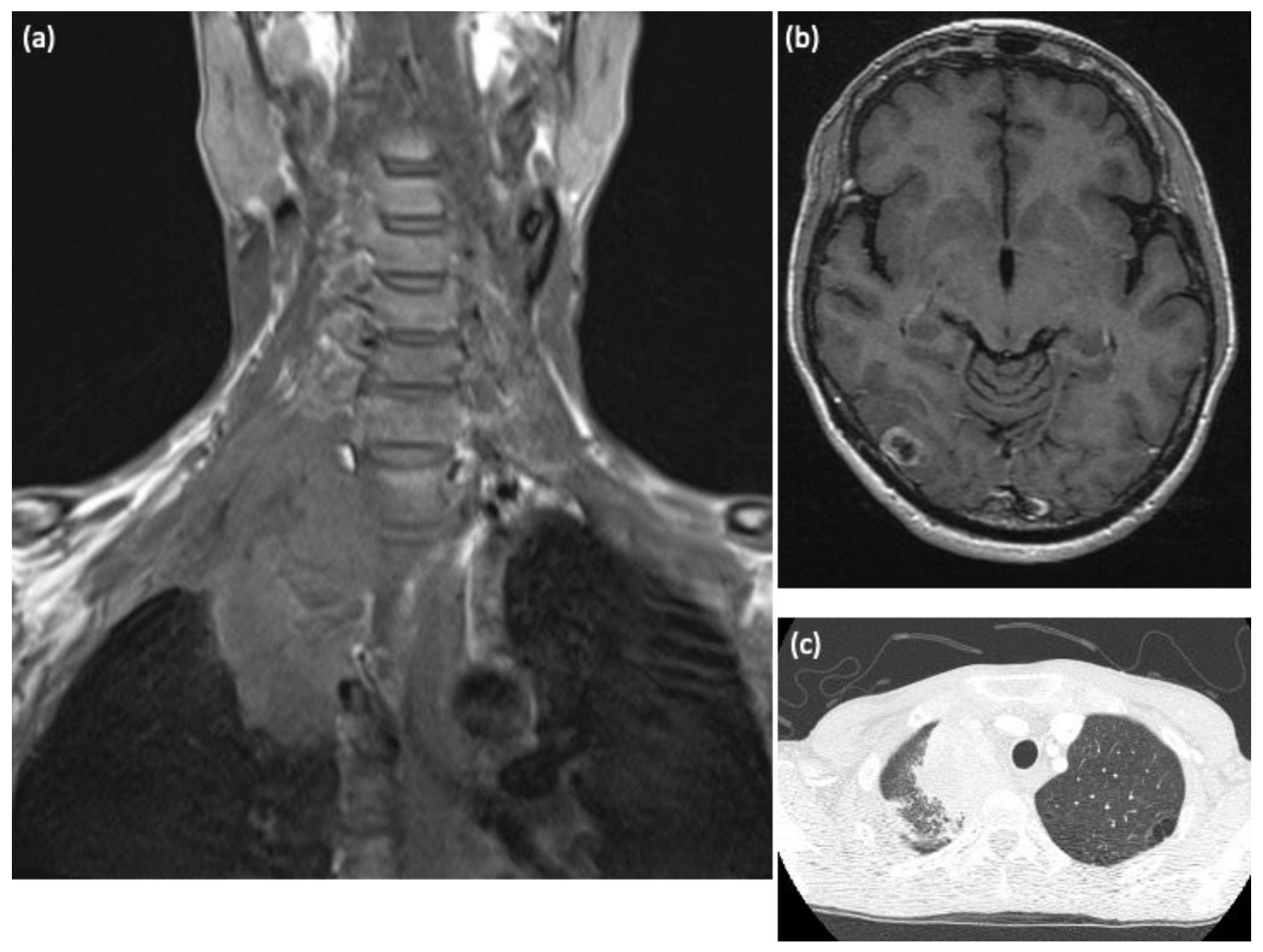

Figure 1

Clinical imaging. (a) Magnetic resonance imaging (MRI) revealed a mass in the right lung apex with extension to the mediastinum and thoracic vertebra. (b) Gadolinium-enhanced MRI revealed a mass with ring enhancement in the right occipital lobe. (c) A chest computed tomography (CT) revealed a mass in the right lung apex and bullae. 

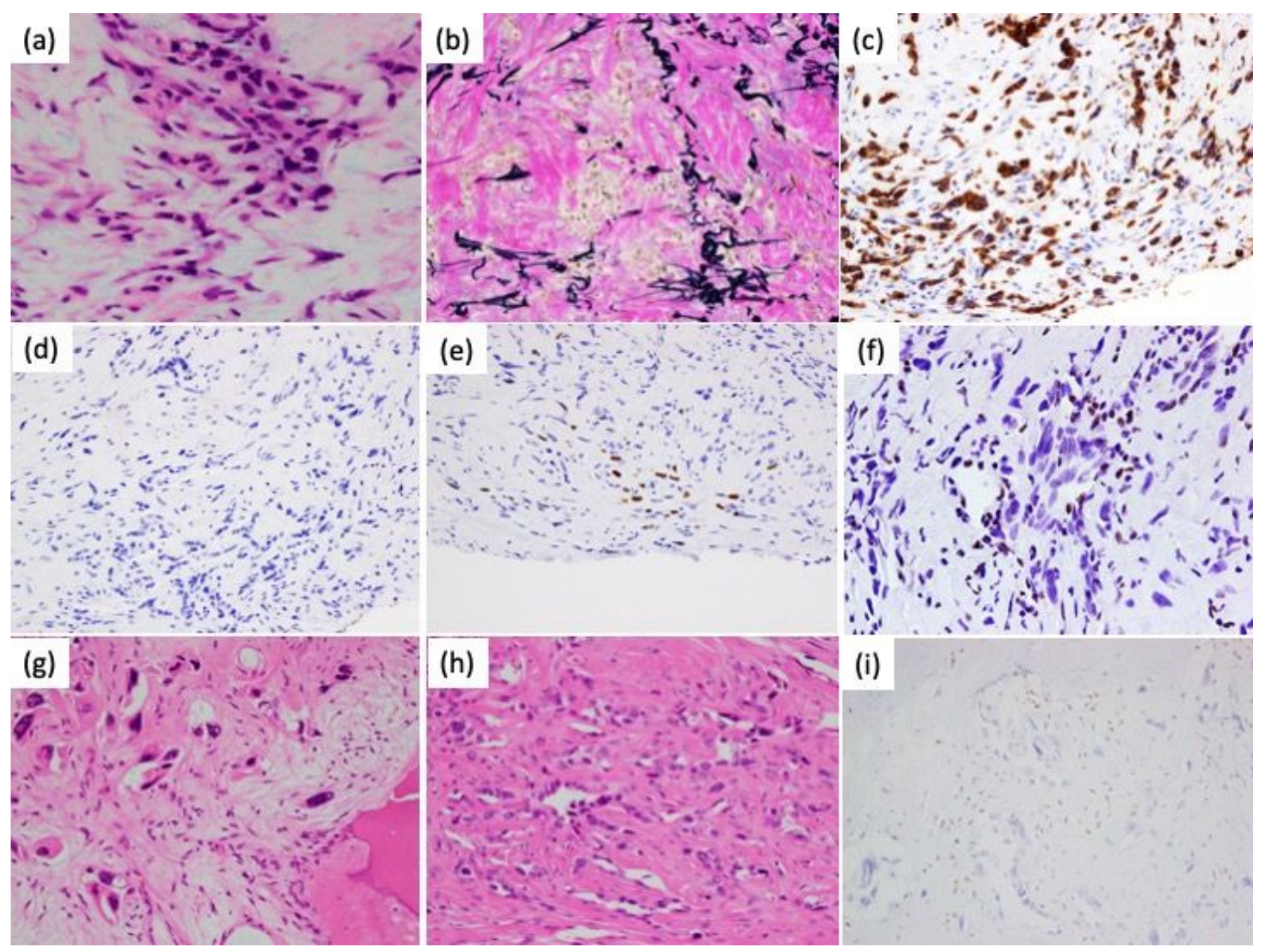

(i)

\section{Figure 2}

Histology and immunohistochemical findings in the thoracic tumor. CT-guided needle biopsy (a-f) and the vertebral tumor during emergency decompression ( $g-i)$. (a) Irregular proliferation of noncohesive spindle or polygonal cells was found with an occasional trabecular pattern. (b) The neoplastic cells infiltrated the lung parenchyma (Elastica van Gieson). (c) Cytokeratin (CK) AE/AE3 immunostaining. Only diffuse staining was observed. (d) Thyroid transcription factor 1 (TTF-1) immunostaining, which was negative in all samples. (e) p40 immunostaining. A few positive nuclei were seen. (f) Brahma-related gene 1 (BRG1) immunostaining. Irregular and larger nuclei were negative, but some small nuclei stained brown, which suggests the loss of BRG1 expression in neoplastic cells. (g) Bizarre, dyscohesive large cells with prominent nuclear atypia and small vesicles were found in the hyalinous stroma. (h) An alveolar pattern was occasionally observed in the vertebral specimen. (i) BRG1 immunostaining. Irregular and larger nuclei in an alveolar arrangement were negative, but some small round and spindle nuclei were stained brown, which indicates the loss of BRG1 expression in also vertebral neoplastic cells. 


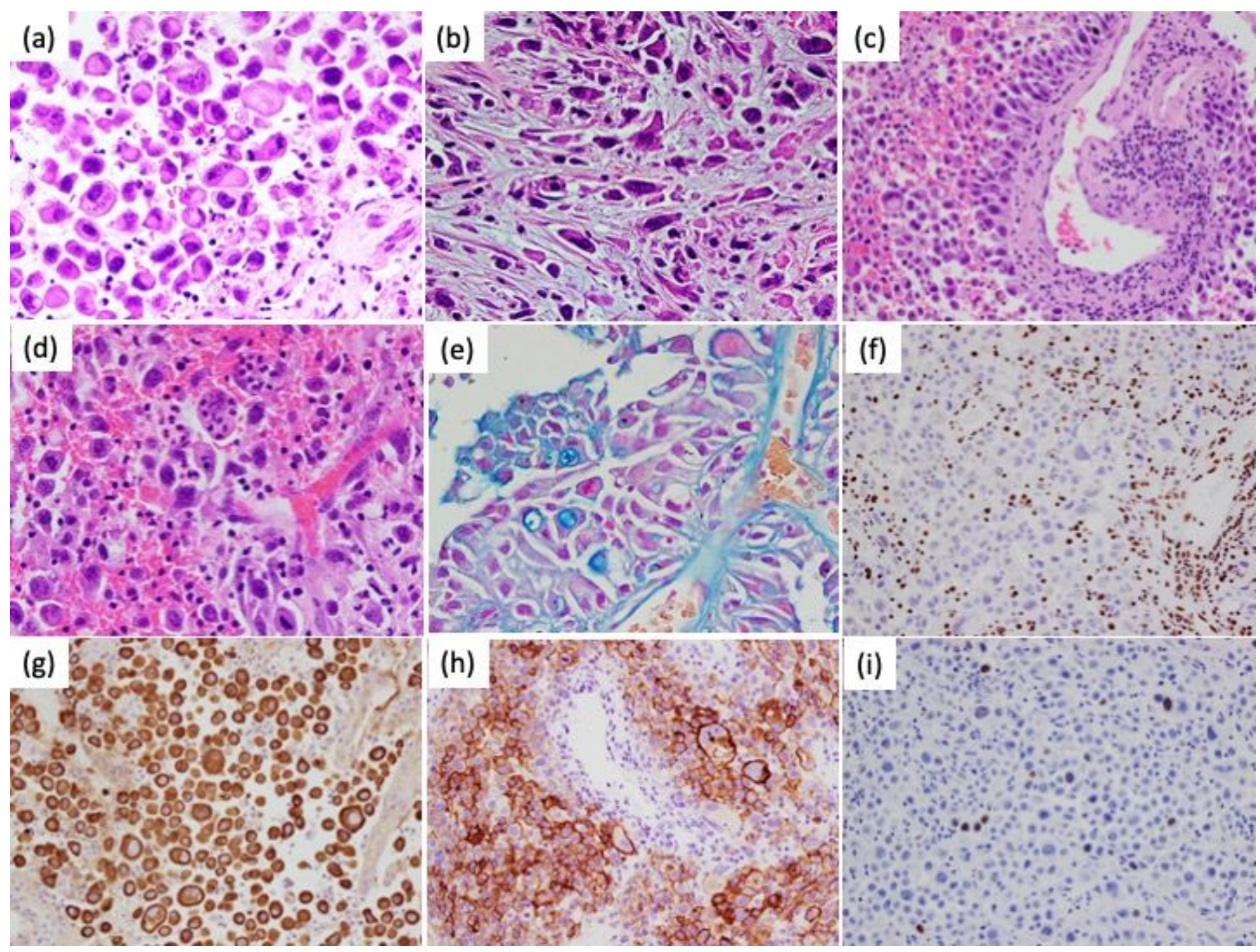

Figure 3

Histology and immunohistochemical findings in the brain metastasis. (a) Large, irregular, and dyscohesive round or polygonal cells exhibited a solid growth pattern. (b) Spindle or polygonal cells were seen in the myxoid stroma. (c) Pseudo-papillary architecture, hobnail cells, and rhabdoid-like cells with eccentric nuclei were observed. (d) The emperipolesis associated with granulocytes was found in large cells (e) Blue positive staining was seen in the cytoplasm of atypical cells, which suggests the presence of intracytoplasmic mucin or small glandular structures (Alcian blue staining). (f) Brahma-related gene 1 (BRG1) immunostaining. Irregular and larger round nuclei were negative, but some small nuclei were stained brown, which suggests the loss of BRG1 expression in these neoplastic cells. (g) Cytokeratin (CK) AE/AE3 immunostaining for which diffuse staining in larger cells was observed. (d) Claudin-4 immunostaining. Staining in the cellular membrane was observed in large cells. (i) p40 immunostaining. A few positive nuclei were seen in large cells. 


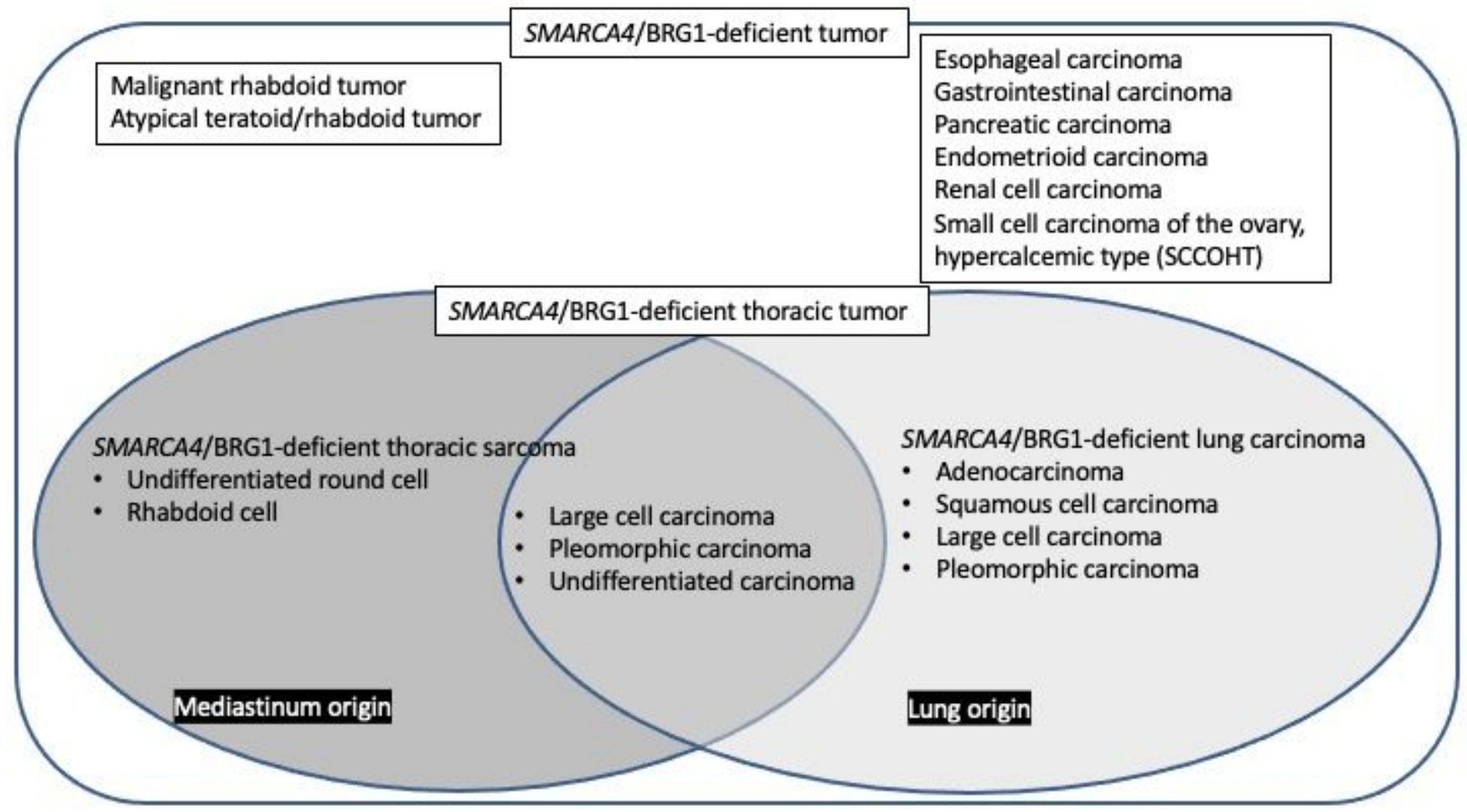

Figure 4

SMARCA4/BRG1-deficient thoracic NSCLC and SMARCA4/BRG1-deficient thoracic sarcoma frequently occur in both the lung and mediastinum. The discrimination of these tumors is difficult because of several clinicopathological similarities.

\section{Supplementary Files}

This is a list of supplementary files associated with this preprint. Click to download.

- CAREchecklist.pdf 